\title{
Der TARMED funktioniert gut, er muss aber aktualisiert werden
}

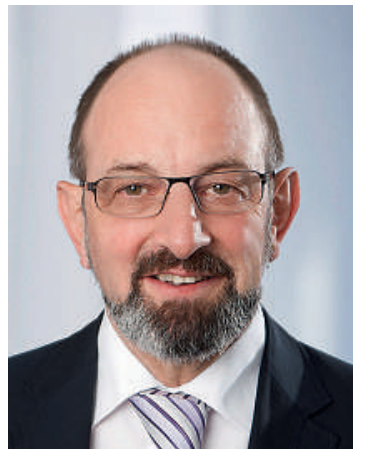

Der vielgeschmähte TARMED wird zu Unrecht verantwortlich gemacht für die Probleme bei der Abgeltung der ambulanten Arztleistungen im schweizerischen Gesundheitswesen. Der Einzelleistungstarif erweist sich bei einer fundierten und umfassenden Analyse durchaus als sachgerecht und alltagstauglich. Dringend notwendig ist jedoch, die Tarifstruktur zu aktualisieren und sie an die heutigen Gegebenheiten anzupassen. Eine kontinuierliche Überarbeitung des TARMED wurde seit seiner Einführung im Jahr 2004 vernachlässigt und hat zu oft überbewerteten Problemen geführt. Das Projekt TARVISION der FMH in Zusammenarbeit mit H+ zeigt, dass die Tarifstruktur technisch problemlos jährlich aktualisiert werden kann, so dass sie den heutigen Anforderungen an einen modernen, adäquaten und alltagstauglichen Einzelleistungstarif genügt. Voraussetzung für eine sachgerechte Revision des Tarifs ist eine Aktualisierung der Kostenmodelle an die aktuellen betriebswirtschaftlichen Gegebenheiten.

\section{Die Tarifstruktur muss sachgerecht sein, und die Berechnung der Entschädigung betriebswirtschaft- lich erfolgen.}

Das Krankenversicherungsgesetz schreibt in Art. 43 Abs. 4 vor, dass eine Tarifstruktur sachgerecht zu sein und die Berechnung der resultierenden Entschädigung betriebswirtschaftlich zu erfolgen hat. Um diese Vorgaben dauerhaft erfüllen zu können, müssen veränderte Rahmenbedingungen sowohl im Bereich der Kostenmodelle wie auch im Bereich des medizinischen Fortschritts kontinuierlich in die bestehende Tarifstruktur einfliessen. Bei der Tarifstruktur TARMED hat eine solche laufende Aktualisierung bisher nicht stattgefunden. Die hinterlegten Daten stammen aus den Jahren 1994 bis 1996 und sind veraltet. Die Abgeltung für die ärztlichen und technischen Leistungen ist darum heute nicht mehr sachgerecht und hat zu schleichenden Einkommensverlusten geführt.

Unter den Tarifpartnern FMH, H+, MTK und santésuisse herrscht Konsens, dass die Tarifstruktur aktualisiert und revi- diert werden muss. Es bestehen jedoch Differenzen im Hinblick auf die konkrete Umsetzung der Revision. Santésuisse blockiert seit Jahren mit der unangebrachten Forderung nach Taxpunktneutralität in der Tarifstruktur und unter missbräuchlicher Anwendung des Einstimmigkeitsprinzips jegliche Entwicklung.

\section{Die Tarifpartner sind eingeladen, sich am Projekt TARVISION der FMH zu beteiligen.}

Zudem hat die eidgenössische Finanzkontrolle EFK zwischen 2008 und 2010 eine Evaluation des TARMED-Tarifs durchgeführt und ist zum Schluss gekommen, dass bei einer Nichteinigung der Tarifpartner der Bund mehr Kompetenzen und Eingriffsmöglichkeiten in die Tarifstruktur erhalten müsse. Allerdings hat die EFK bei den Schlussfolgerungen unterschlagen, dass sechs von elf der von ihr überprüften Fallbeispiele untertarifiert sind. Die Fallbeispiele betreffen vorwiegend Hausärzte und machen über $50 \%$ des TARMEDUmsatzes aus. Trotzdem hat das Parlament eine Gesetzesänderung gutgeheissen, die es dem Bundesrat erlaubt, Anpassungen an der Tarifstruktur vorzunehmen, wenn sich die Struktur als nicht mehr sachgerecht erweist.

Aufgrund dieser unhaltbaren Situation hat sich die FMH bereits im Herbst 2010 entschlossen, die Revision der Tarifstruktur mit TARVISION eigenständig an die Hand zu nehmen. Seither haben die FMH sowie Experten unterschiedlicher Disziplinen und unter Einbezug der Fachgesellschaften die Revision vorangetrieben und bereits wichtige Meilensteine erreicht. Ziel ist es, die Kostenmodelle (z.B. nichtärztliche Löhne) zu aktualisieren und Unwuchten (z.B. Anpassungen der Minutagen) des Tarifs zu beheben. Die bestehenden Modelle werden lediglich aktualisiert. In der kürzlich von $\mathrm{H}+$ und FMH unterzeichneten Absichtserklärung wird die gemeinsame Zusammenarbeit und Koordination der Revisionsprojekte für eine betriebswirtschaftliche und adäquate Abgeltung der ambulanten Leistungen der Arztpraxen sowie der ambulanten Spitalleistungen bekräftigt (weitere Informationen auf www.fmh.ch $\rightarrow$ Tarife $\rightarrow$ TARMED Tarif). Wir laden alle Tarifpartner ein, sich aktiv in das Projekt TARVISION einzubringen.

Dr. med. Ernst Gähler, Mitglied des Zentralvorstandes, Verantwortlicher Ambulante Tarife und Verträge Schweiz 